\title{
Time and Frequency Synchronization for Hiperlan/2
}

\author{
Anna Berno ${ }^{1}$ and Nicola Laurenti ${ }^{2}$ \\ 1 Dipartimento di Elettronica ed Informatica, Università di Padova \\ 35131 Padova, Italy. \\ Now with Fracarro Radioindustrie, 31033 Castelfranco Veneto, Italy \\ 2 Dipartimento di Elettronica ed Informatica, Università di Padova \\ nil@dei.unipd.it \\ http://www.dei.unipd.it/〜nil/index.html
}

\begin{abstract}
The Hiperlan/2 standard [1]-[3] for wireless LAN transmission in the $5 \mathrm{GHz}$ frequency band makes use of OFDM modulation with a TDMA access scheme, in order to efficiently exploit time dispersive channels with frequency selective fading.

It is well known that the performance of OFDM schemes is very sensitive to synchronization: symbol timing and carrier frequency errors must be carefully estimated and corrected at the receiver.

We propose a scheme for time and frequency offset estimation, derived form those presented in [4]-7], suited to all the transmission burst types of the standard. The scheme makes use of the periodic structure of each burst preamble and is robust with respect to distortions induced by dispersive channels.

We evaluate its performance both via statistical analysis and simulation in the presence of AWGN and dispersive channels, and also present an original technique for performance evaluation of the timing synchronization in dispersive environments, based on the cumulative distribution function of the useful signal power after demodulation.
\end{abstract}

\section{Introduction}

The Hiperlan/2 standard [1-3 aims at providing high bit rate wireless links to fixed or portable terminals within a local (mainly indoor) environment, with a channel bandwidth of $20 \mathrm{MHz}$ in the $5 \mathrm{GHz}$ band. It makes use of an OFDM modulation technique with cyclic prefix which makes transmissions very robust to dispersive channel affected by frequency selective fading, thus increasing its spectral efficiency, but is very sensitive to timing and frequency offsets between transmitter and receiver, which can cause intersymbol and intercarrier interference in the demodulated signal. It is therefore mandatory that time and frequency offsets be carefully estimated and corrected at the receiver.

We present a time and frequency synchronization scheme derived from [4][7], based on periodic preambles and adapted to suit Hiperlan/2 burst types. Since it is based on the signal periodicity rather than its actual expression, and

E. Gregori et al. (Eds.): NETWORKING 2002, LNCS 2345, pp. 491-502 2002.

(C) Springer-Verlag Berlin Heidelberg 2002 
periodicity is preserved through the channel, the algorithm effectively can work also in dispersive environment. Its performance is evaluated both in AWGN and dispersive channels by means of statistical analysis and simulation results. In particular we introduce an original method to evaluate the time synchronization performance in the case of a dispersive channel in terms of the power of the useful component in the demodulated signal.

The paper is organized as follows. In Section 2 we set up the system model with time and frequency offsets and a non ideal channel and in Section 3 we discuss the effects of time and frequency offsets on the performance of OFDM systems in an ideal or dispersive channel. In Section 4 we present the estimation algorithms and the techniques for analytical evaluation of their performance. Section [5]collects and discusses results obtained from simulations together with those derived analytically. Eventually we draw conclusions in Section 6.

\section{System Model}

OFDM parameters for Hiperlan/2 [3] are summarized in Table 1] The OFDM symbols are concatenated into the payload; a preamble is preponed to the payload and the two together form a physical layer (PHY) burst. Five different PHY burst types are provided [3], each corresponding to a different transmission mode: broadcast, downlink, uplink with short preamble, uplink with long preamble, direct link (optional). The preambles structure is illustrated in Fig. 1]

Table 1. OFDM parameters for Hiperlan/2

\begin{tabular}{|l|l|}
\hline Parameter & Value \\
\hline Sampling rate $F_{0}=1 / T$ & $20 \mathrm{MHz}$ \\
Carrier central frequency $f_{\mathrm{c}}$ & $5.2 \mathrm{GHz}$ \\
FFT size $N$ & 64 \\
Useful symbol part duration $T_{\mathrm{U}}$ & $64 T=3.2 \mu \mathrm{s}$ \\
Cyclic prefix duration $T_{\mathrm{CP}}$ & $16 T=0.8 \mu \mathrm{s}$ (optional $8 T=0.4 \mu \mathrm{s})$ \\
Symbol interval $T_{\mathrm{S}}=T_{\mathrm{U}}+T_{\mathrm{CP}}$ & $80 T=4.0 \mu \mathrm{s}$ (optional $72 T=3.6 \mu \mathrm{s})$ \\
Number of data sub-carriers $N_{\mathrm{SD}}$ & 48 \\
Number of pilot sub-carriers $N_{\mathrm{SP}}$ & 4 \\
Total number of sub-carriers $N_{\mathrm{ST}}=N_{\mathrm{SD}}+N_{\mathrm{SP}}$ & 52 \\
Sub-carrier spacing $F=1 / T_{\mathrm{U}}$ & $0.3125 \mathrm{MHz}$ \\
Nominal bandwidth $B=N_{\mathrm{ST}} F$ & $16.25 \mathrm{MHz}$ \\
Data symbol constellations & BPSK, QPSK, 16-QAM, 64-QAM \\
\hline
\end{tabular}

Relatively to transmission, the system can be modeled as in Fig. 22 Data and pilot symbols modulate the $N_{\mathrm{ST}}$ active subcarriers with indices in $\mathcal{M}=$ $\left\{-N_{\mathrm{ST}} / 2, \ldots,-1,1, \ldots, N_{\mathrm{ST}} / 2\right\}$, giving the modulated signal

$$
s(t)=\sum_{m \in \mathcal{M}} \sum_{l=-\infty}^{+\infty} S_{m}\left(l T_{\mathrm{S}}\right) p\left(t-l T_{\mathrm{S}}\right) e^{j 2 \pi m F\left(t-l T_{\mathrm{S}}\right)}
$$




\begin{tabular}{|c|c|c|c|c|c|c|c|c|c|c|c|c|c|c|c|}
\hline & $\mathrm{Sec}$ & tio & $\mathrm{n} A$ & & & Sec & tio & $\mathrm{a} \mathrm{E}$ & & & & tiol & & & \\
\hline $5 x$ & $\times 16$ & $6 T$ & $=4$ & $\mu \mathrm{s}$ & 5 & $\times 1$ & $3 T$ & $=$ & $\overrightarrow{\mu \mathrm{S}}$ & & $5 \times$ & $2 T$ & $8 \mu \mathrm{s}$ & & \\
\hline $\mathrm{A}$ & $-\mathrm{A}$ & $\mathrm{A}$ & $-\mathrm{A}$ & $-\mathrm{A}$ & B & $\mathrm{B}$ & B & B & $-B$ & $\mathrm{C}^{\prime}$ & $\mathrm{C}^{\prime \prime}$ & $\mathrm{C}^{\prime}$ & $\mathrm{C}^{\prime \prime}$ & $\mathrm{C}^{\prime}$ & broadcast \\
\hline & & & & & & & & & & $\mathrm{C}^{\prime}$ & $\mathrm{C}^{\prime \prime}$ & $\mathrm{C}^{\prime}$ & $\mathrm{C}^{\prime \prime}$ & $\mathrm{C}^{\prime}$ & downlink \\
\hline & & & & & $\mathrm{B}$ & B & $\mathrm{B}$ & $\mathrm{B}$ & $-\mathrm{B}$ & $\mathrm{C}^{\prime}$ & $\mathrm{C}^{\prime \prime}$ & $\mathrm{C}^{\prime}$ & $\mathrm{C}^{\prime \prime}$ & $\mathrm{C}^{\prime}$ & uplink short \\
\hline $\mathrm{B}$ & $\mathrm{B}$ & $\mathrm{B}$ & B & $\mathrm{B}$ & $\mathrm{B}$ & B & $\mathrm{B}$ & $\mathrm{B}$ & $-\mathrm{B}$ & $\mathrm{C}^{\prime}$ & $\mathrm{C}^{\prime \prime}$ & $\mathrm{C}^{\prime}$ & $\mathrm{C}^{\prime \prime}$ & $\mathrm{C}^{\prime}$ & uplink long \\
\hline $\mathrm{B}$ & $\mathrm{B}$ & B & B & B & B & B & B & B & $-\mathrm{B}$ & $\mathrm{C}^{\prime}$ & $\mathrm{C}^{\prime \prime}$ & $\mathrm{C}^{\prime}$ & $\mathrm{C}^{\prime \prime}$ & $\mathrm{C}^{\prime}$ & direct link \\
\hline
\end{tabular}

Fig. 1. Structure of Hiperlan/2 preambles for all burst types.

with $p(t)$ the rectangular window on the interval $\left[-T_{\mathrm{CP}}, T_{\mathrm{U}}\right)$ and $S_{m}$ the transmitted complex symbols.

The channel impulse response and the additive noise can be replaced by their baseband equivalents. The former can be modeled as a tapped delay line [9], with non-uniform time spacing between taps and an exponentially decaying power delay profile. A Doppler spread of $52 \mathrm{~Hz}$ is assumed for each tap, corresponding to a terminal speed of $3 \mathrm{~m} / \mathrm{s}$, so that the channel coherence time results $\tau_{c} \simeq 20$ ms, while each burst is always shorter than $2 \mathrm{~ms}$. The channel can therefore be considered time-invariant for the duration of a burst and its impulse response is written as

$$
h(\tau)=\sum_{k=0}^{N_{h}-1} a_{s} \delta\left(\tau-\tau_{s}\right)
$$

where $\tau_{s}$ are the delays, and $a_{s}$ the complex amplitudes. BRAN defined five channel models for Hiperlan/2 simulations: A and B, with a delay spread shorter than the duration of the cyclic prefix; C, D and E, with longer delay spread 8.

With $w(\cdot)$ the baseband equivalent noise, the received signal before sampling is $y(t)=[s * h(t)] e^{j 2 \pi \Delta t}+w(t)$, with $\Delta f=f_{0}-f_{0}^{\prime}$ the carrier frequency offset between transmitter and receiver. If the starting instant of the FFT demodulating window is at $t_{0}$ we can then write the demodulated signal as

$$
Y_{m}\left(k T_{\mathrm{S}}\right)=\sum_{n=0}^{N-1} y\left(t_{0}+k T_{\mathrm{S}}+n T\right) e^{-j 2 \pi m n / N} .
$$

The mismatch that can possibly turn up between the transmitter and receiver oscillators in the forms of carrier frequency offset, phase noise in the RF oscillators, sampling or clock frequency offset, sampling or clock jitter, or OFDM symbol timing error, is a common cause of impairment in an OFDM system [10].

In the course of our work only carrier frequency offset and symbol timing error will be considered with reference to a Hiperlan/2 system. As regards sampling frequency errors, it has to be noted that a quantification of their effect leads 

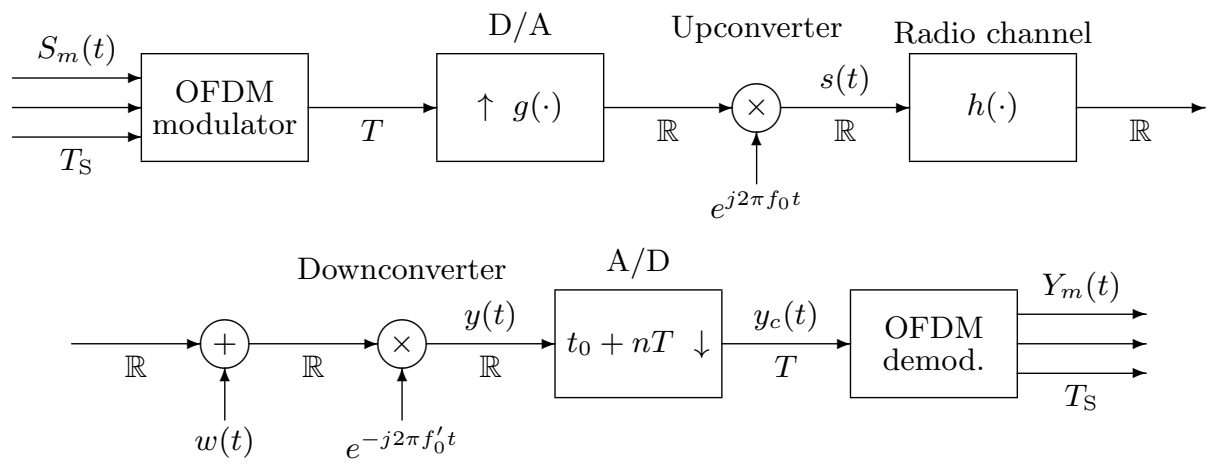

Fig. 2. Simplified model of an OFDM system with time and frequency offsets

to the conclusion that the maximum symbol phase rotation that can affect a Hiperlan/2 system approximates about 0.117 degrees if we consider an oscillator with a frequency instability of $10 \mathrm{ppm}$. As a consequence, the effects induced on the system by a sampling frequency offset have been neglected in our work.

\section{$3 \quad$ Effects of Time and Frequency Offsets}

\subsection{Carrier Frequency Offset}

A visible effect of a carrier frequency offset $\Delta f[4]$ on the received symbols is a rotation of the received constellation of a phase equal to $2 \pi t \Delta f, t \in Z\left(T_{\mathrm{S}}\right)$. Due to $\Delta f$ a shift of the received spectrum on the frequency axis and consequently a loss of mutual orthogonality between the subcarriers occurs. This results in InterChannel Interference (ICI). The variance of the ICI process, $\sigma_{\mathrm{ICI}_{k}}^{2}$, is the sum of the variances of the interference contributions:

$$
\sigma_{\mathrm{ICI}_{k}}^{2}=\sum_{m \neq k} \sigma_{S_{m}}^{2}\left|H\left(f_{m}\right)\right|^{2} \cdot \operatorname{sinc}^{2}\left(f_{m}-f_{k}+\Delta f\right),
$$

with $H(f)$ the channel frequency response. The statistical properties of the ICI were evaluated in [10] showing that, contrary to what stated in [4] a Gaussian distribution can not be assumed in general, but it represents a fair approximation for dense constellations and small $\Delta f$.

\subsection{Symbol Timing}

A shift of the FFT observation window at the receiver with respect to the transmission window exceeding the guard interval causes samples from the previous or following OFDM symbol to fall within the current window (resulting in InterSymbol Interference, ISI) and samples of the useful part of the current symbol to 
be lost (resulting in loss of orthogonality among the subchannels and thus ICI). The optimum positioning of the observation window should take advantage of the margin left by the presence of the cyclic prefix. This margin is reduced, however, by a time-dispersive channel, because the first samples of the prefix and the last samples of the data might suffer from the interference due to delayed or early replicas of the adjacent OFDM symbols. On the other hand, ICI and ISI are unavoidable if the channel impulse response length exceeds the guard interval length.

In the presence of a time-dispersive channel $h(t)$, the input to the demodulating FFT window will in general contain some interference from adjacent OFDM symbols. Correspondingly to the channel frequency response at frequency $m F$, $H(m F)$, the demodulated symbol on the $m$-th subcarrier will be therefore affected by an amplitude and phase distortion, as well as by ICI and ISI. In this case, since the channel distortion and the consequent need for amplitude and phase equalization are unavoidable, finding the optimum timing means choosing the starting instant for the FFT demodulation window such that the power of the useful component of the signal is maximized. If we neglect noise, each output signal $Y_{m}(t)$ can be written as:

$$
\begin{aligned}
Y_{m}\left(k T_{\mathrm{S}}\right)= & \Phi_{m, m}\left(k T_{\mathrm{S}}, k T_{\mathrm{S}}\right) S_{m}\left(k T_{\mathrm{S}}\right)+\sum_{r \neq m} \Phi_{r, m}\left(k T_{\mathrm{S}}, k T_{\mathrm{S}}\right) S_{r}\left(k T_{\mathrm{S}}\right) \\
& +\sum_{l \neq k} \sum_{r} \Phi_{r, m}\left(l T_{\mathrm{S}}, k T_{\mathrm{S}}\right) S_{r}\left(l T_{\mathrm{S}}\right),
\end{aligned}
$$

with

$$
\Phi_{r, m}\left(l T_{\mathrm{S}}, k T_{\mathrm{S}}\right)=\frac{1}{N} \sum_{n=0}^{N-1} \mathrm{~W}_{N}^{-n m} \int_{-T_{\mathrm{CP}}}^{T_{\mathrm{U}}} h\left(t_{0}+n T+k T_{\mathrm{S}}-l T_{\mathrm{S}}-v\right) e^{j 2 \pi r F v} d v .
$$

The second and third term of (5) represent the interference (respectively ICI and ISI) experienced by the demodulated OFDM symbol. On the other hand, under the simplifying assumption that $S_{r}\left(l T_{\mathrm{S}}\right)$ are i.i.d. QAM symbols, with $\mathrm{E}\left[S_{m}\right]=0$ and $\mathrm{E}\left[\left|S_{m}\right|^{2}\right]=M_{S}$, the term $M_{\mathrm{u}}(m, k)=M_{S}\left|\Phi_{m, m}\left(k T_{\mathrm{S}}, k T_{\mathrm{S}}\right)\right|^{2}$ is the power of the useful signal component with the FFT window starting at $t_{0}$. Consider the multipath channel impulse response 2, $M_{\mathrm{u}}$ can be evaluated as:

$$
M_{\mathrm{u}}=\frac{M_{S}}{N^{2}}\left|\sum_{s=0}^{N_{h}-1} a_{s} \gamma\left(\tau_{s}-t_{0}\right) e^{-j 2 \pi m F \tau_{s}}\right|^{2}
$$

with

$$
\gamma(t)= \begin{cases}N+\lceil t / T\rceil & -T_{\mathrm{U}}<t \leq-T \\ N & -T<t \leq T_{\mathrm{CP}} \\ N-\lceil t / T\rceil+N_{\mathrm{CP}} & T_{\mathrm{CP}}<t<T_{\mathrm{S}} \\ 0 & \text { elsewhere }\end{cases}
$$

The quantity $M_{\mathrm{u}}$ can be used to evaluate the symbol timing estimators performance in the presence of a time-dispersive channel. If $M_{\mathrm{u}}$ is normalized to $|H(m F)|^{2}$, we obtain $\bar{M}_{\mathrm{u}}$. Therefore, the closer $\bar{M}_{\mathrm{u}}$ approaches unity, the better timing estimation is performed. 


\section{Synchronization Algorithms}

Synchronization methods for OFDM can follow either a pilot-aided or a blind approach. The presence of burst preambles in the transmitted Hiperlan/2 signal calls for the former approach, that is generally faster, while the latter relies on long range signal statistics.

In turn, pilot-aided estimation can be accomplished at the receiver either in the time domain (operating on the received signal prior to FFT demodulation), or in the frequency domain (i.e. operating on the demodulated signal). However, since the A and B preamble sections do not exhibit the cyclic prefix structure, the demodulated signal would suffer from loss of orthogonality in the presence of an even slightly dispersive channel, which makes frequency domain techniques less viable. Among time-domain methods, the repetitive structure of the preambles suggests that an algorithm such as the one proposed by Hanzo and Keller [4] can be modified to be adapted to Hiperlan/2 preambles, to achieve frequency and timing estimation without knowledge of the adopted reference sequence. Likewise, it is possible to think of the sections that compose all the preambles as if they were made by two identical symbols in the time domain, as required by Schmidl and Cox algorithm [6]-7].

\subsection{Symbol Timing Estimation}

The Schmidl and Cox algorithm is modified considering that the received preamble signal $r(t)$ keeps its periodic structure, with period $T_{p}=L T$, inside the observation interval $(L+C) T$, that is $r_{m+L}=r_{m}$ for $m=m_{0}, \ldots, m_{0}+C$. The timing metric for symbol synchronization $M(d)$, proposed in [6], is modified as

$$
M(d)=\frac{\sum_{m=0}^{C_{1}-1} r_{d+m}^{*} r_{d+m+L}+\sum_{m=C_{1}}^{C_{1}+C_{2}-1} r_{d+m}^{*}\left(-r_{d+m+L}\right)}{\sum_{m=0}^{C-1}\left|r_{d+m+L}\right|^{2}} .
$$

Expression (9) can be applied to all Hiperlan/2 burst preambles with different values for the parameters, correlates samples at a distance $L T$ within a sliding window of length $(C+L) T$, thus overcoming the restriction imposed in [6] on the window length, which could include only one OFDM symbol. The optimum timing $d_{\text {opt }}$ can be found by maximizing the metric (9): the parameter values for each burst type are given in Table 2

On an ideal channel the performance of the timing estimator can be assessed in terms of its probability mass distribution, as $d_{\mathrm{opt}}$ is a discrete variable. With a dispersive channel, however, it is more appropriate to evaluate the power of the useful signal component (7) obtained with the estimated correct positioning of the FFT window.

\subsection{Carrier Frequency Offset Estimation}

Making use of the optimal timing information, frequency synchronization can be performed as if no timing error affected the system. Moreover, following the hint 
given in [4]- 5], an important effect can be achieved: the use of a training sequence with a shorter periodicity allows the frequency offset estimation range to be widened. Each OFDM symbol of Hiperlan/2 preambles is composed of $T_{\mathrm{U}} / T_{p^{\prime}}=$ $K$ short symbols, which can be considered identical except for a sign inversion, they have a minimum period of length $T_{p^{\prime}}=T_{\mathrm{U}} / K$. The carrier frequency offset is estimated as:

$$
\widehat{\Delta f}=\frac{1}{2 \pi L T} \arg \left(\sum_{m=0}^{L-1} \alpha r_{m+d_{\mathrm{opt}}} r_{m+d_{\mathrm{opt}}+L}^{*}\right),
$$

whit $d_{\text {opt }}$ the optimum symbol timing and $\alpha$ and $L$ depend on the preamble structure and are given in Table 2 The maximum frequency offset that can be detected becomes $\Delta f_{\max }=1 /(2 L T)=N F /(2 L)$. As an example, for the broadcast burst, the initial frequency offset required is $\Delta f_{\max }=0.625$ which yields an upper bound to the oscillators stability of about 120 parts per million (ppm), a condition easily met by commercial devices.

Table 2. Parameters of the symbol timing and carrier frequency offset estimators for the different burst types

\begin{tabular}{|l|c|cccc|ccc|}
\hline & & \multicolumn{4}{|c|}{ Symbol timing } & \multicolumn{4}{c|}{ Frequency offset } \\
Burst type & Preamble section & $L$ & $C_{1}$ & $C_{2}$ & $C$ & $\alpha$ & $L$ & $\Delta f_{\max }$ \\
\hline Broadcast & $\mathrm{A}$ & 32 & 32 & 0 & 32 & -1 & 16 & $2 F=0.625 \mathrm{MHz}$ \\
Downlink & $\mathrm{C}$ & 64 & 96 & 0 & 96 & 1 & 64 & $F / 2=0.156 \mathrm{MHz}$ \\
Uplink short & $\mathrm{B}$ & 32 & 32 & 0 & 32 & 1 & 16 & $2 F=0.625 \mathrm{MHz}$ \\
Uplink long & $\mathrm{B}$ & 80 & 64 & 16 & 80 & 1 & 16 & $2 F=0.625 \mathrm{MHz}$ \\
Direct link & $\mathrm{B}$ & 80 & 64 & 16 & 80 & 1 & 16 & $2 F=0.625 \mathrm{MHz}$ \\
\hline
\end{tabular}

The statistical evaluation of the frequency offset estimator can be made in terms of its conditional mean and statistical power, that is $\mathrm{E}[\widehat{\Delta f} \mid \Delta f]$ and $\mathrm{E}\left[\widehat{\Delta f}^{2} \mid \Delta f\right]$. Our analysis is brought on in the hypothesis of perfect symbol timing correction. The received signal $r(n T)$ can be written as $r_{n+d_{\text {opt }}}=$ $s_{n} e^{j 2 \pi n T \Delta f}+w_{n}$, with $s_{n}$ the transmitted OFDM signal and $w_{n} \in \mathcal{N} C\left(0,2 \sigma^{2}\right)$ complex gaussian noise. With the simplifying hypothesis of high signal to noise ratio we can write

$$
\sum_{m=0}^{L-1} r_{m}^{*} r_{m+L} \simeq \sum_{m}\left|s_{m}\right|^{2}+\sum_{m}\left(s_{m}^{*} \eta_{m+L}+s_{m} \eta_{m}^{*}\right)=S+W
$$

with $S=\sum_{m}\left|s_{m}\right|^{2}$ and $W \in \mathcal{N} C\left(0,4 \sigma^{2} S\right)$. Approximating (10) with $\widehat{\Delta f}=$ $\arg (S+W) /(2 \pi L T)$ and following [11] the conditional PDF of $\widehat{\Delta f}$ for $\lambda \in$ $\left[-\frac{1}{2 L T}, \frac{1}{2 L T}\right]$ is:

$$
f_{\widehat{\Delta f} \mid \Delta f}(\lambda \mid \mu)=2 \pi L T\left(c_{0}+\sum_{n=1}^{+\infty} c_{n} \cos n 2 \pi L T(\lambda-\mu)\right) .
$$


with $\rho=S^{2} / \mathrm{E}\left[|W|^{2}\right]=L\left\langle\left|s_{m}\right|^{2}\right\rangle / 2 \mathrm{E}\left[\left|w_{m}\right|^{2}\right]$, where $\left\langle\left|s_{m}\right|^{2}\right\rangle$ is the mean of the deterministic sequence $\left|s_{m}\right|^{2}$ in the time domain, and

$$
c_{0}=\frac{1}{2 \pi}, c_{n}=\frac{1}{2 \sqrt{\pi}} \sqrt{\rho} e^{-\frac{\rho}{2}}\left[I_{\frac{n-1}{2}}\left(\frac{\rho}{2}\right)+I_{\frac{n+1}{2}}\left(\frac{\rho}{2}\right)\right] .
$$

With a term by term integration we have

$$
\begin{aligned}
\mathrm{E}[\widehat{\Delta f} \mid \Delta f] & =\frac{1}{L T} \sum_{n=1}^{+\infty} \frac{(-1)^{n+1} c_{n}}{n} \sin (n 2 \pi L T \Delta f) \\
\mathrm{E}\left[\widehat{\Delta f}^{2} \mid \Delta f\right] & =\frac{1}{12(L T)^{2}}+\frac{1}{\pi(L T)^{2}} \sum_{n=1}^{+\infty} \frac{(-1)^{n} c_{n}}{n^{2}} \cos (n 2 \pi L T \Delta f) \\
\operatorname{Var}[\widehat{\Delta f} \mid \Delta f] & =\mathrm{E}\left[\widehat{\Delta f}^{2} \mid \Delta f\right]-\{\mathrm{E}[\widehat{\Delta f} \mid \Delta f]\}^{2} .
\end{aligned}
$$

\section{Simulation Results}

Simulations have been carried out for each of the five burst types supported by Hiperlan/2, with an AWGN channel as well as with the channel models in [8], at different SNR conditions. Here we discuss the main results.

\subsection{Synchronization Performance with AWGN Channel}

Symbol Timing Estimation. The results for the symbol timing estimators are illustrated in Fig. 3 in terms of histograms of the estimation error.

Broadcast Burst. The performance of the symbol timing estimator is very good even at low signal to noise ratio, since even with $\mathrm{SNR}=5 \mathrm{~dB}$ the percentage of correct estimations reaches $80 \%$ of the total simulated transmissions.

Downlink Burst. The mass distribution presents an evident bias towards a delay of one or more samples in the timing estimation. In our opinion this is due to the fact that correlation between the samples falling in the two halves of the observation window in use for the calculation of the timing metric periodically happens to be greater when the window is filled with one or more samples of the OFDM symbol that follows the preamble, than when it is correctly placed on the preamble only.

Uplink Burst with Short Preamble. Symbol timing performs well also in the case of an uplink burst with short preamble. Even at SNR $=5 \mathrm{~dB}$ in fact the percentage of correct timing decisions reaches $70 \%$ of the total simulated transmissions and grows to more than $90 \%$ at $\mathrm{SNR}=10 \mathrm{~dB}$.

Uplink Burst with Long Preamble and Direct Link Burst. The performance of the algorithm is extremely good in this case, due to the fact that the observation window is longer than in the other cases, thus reducing the estimation variance. The percentage of correct estimations equals almost $100 \%$ even at $\mathrm{SNR}=5 \mathrm{~dB}$. 
SNR broadcast downlink uplink short uplink long
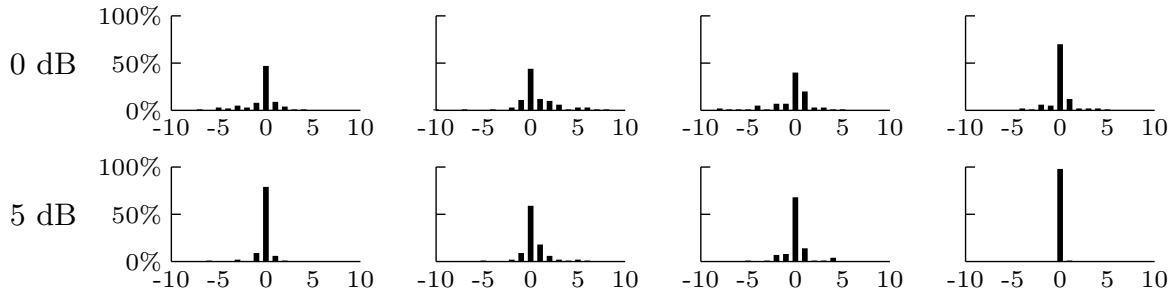

$10 \mathrm{~dB}$


$20 \mathrm{~dB}$
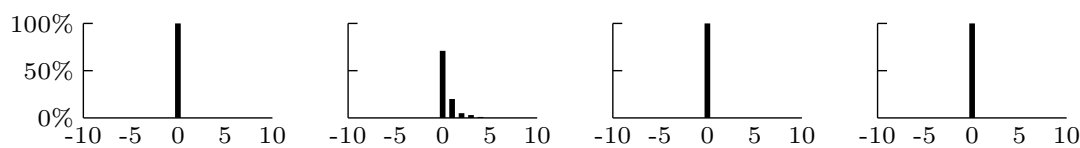

Fig. 3. Histograms of symbol timing estimation error for all burst preambles at different SNR values
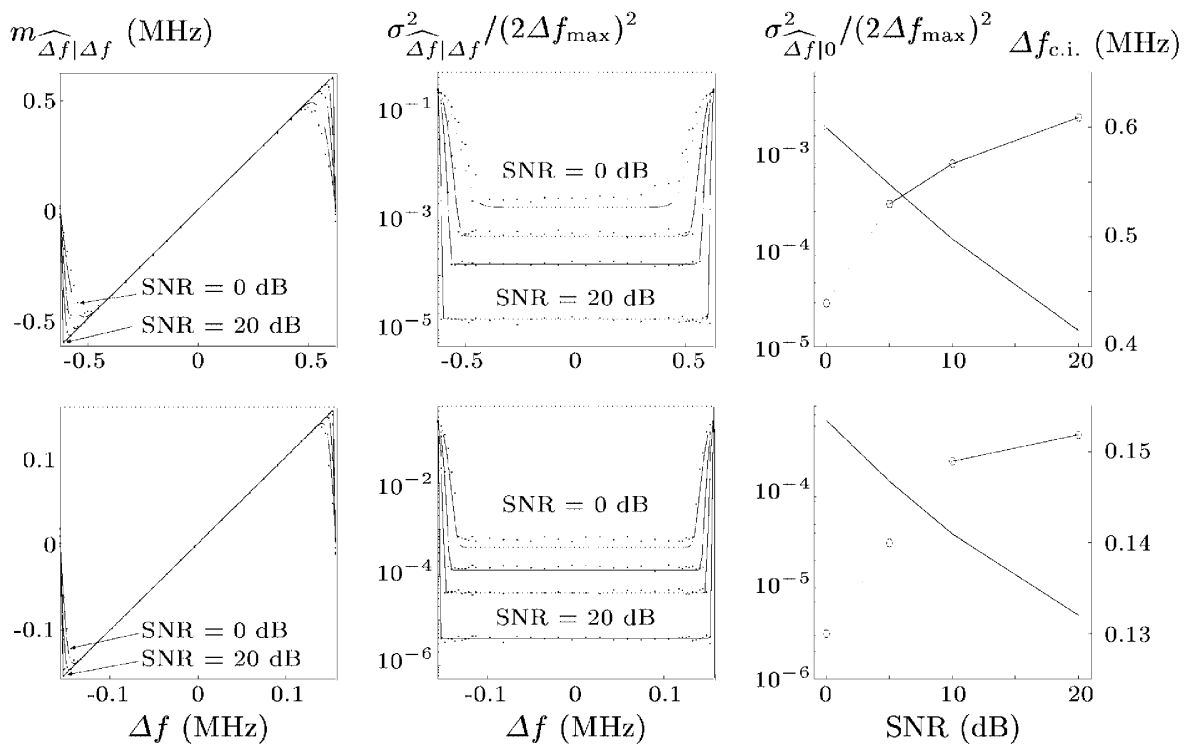

Fig. 4. Statistics of the carrier frequency offset estimator using the broadcast (top) and downlink (bottom) burst preambles. On the left and in the center: conditional mean and normalized conditional variance versus true offset in the range $\left[-\Delta f_{\max }, \Delta f_{\max }\right]$ for $\mathrm{SNR}=0,5,10,20 \mathrm{~dB}$; on the right: normalized variance in the coherence interval (marked with $\times, \log$ scale on the left axis) and width of the coherence interval (marked with $\circ$, linear scale on the right axis) versus channel SNR. 
Carrier Frequency Offset Estimation. The performance of the carrier frequency offset estimator has been evaluated through its mean and variance conditioned on the actual value of the carrier frequency offset $\Delta f$, in the range $\left[-\Delta f_{\max }, \Delta f_{\max }\right]$.

Typically, the estimator can be considered practically unbiased within a range $\left[-\Delta f_{\text {c.i. }}, \Delta f_{\text {c.i. }}\right]$, named coherence interval (c.i.) and within this range, the estimator variance is nearly constant. Outside the c.i. the estimation presents a bias towards the origin and its variance rapidly grows, so that the estimation is clearly not reliable. Therefore, $\Delta f_{\text {c.i. }}$ represents the maximum carrier frequency offset that can reliably be estimated. As the SNR increases, the estimator variance in the c.i. decreases in an inversely proportional fashion, while the width of the c.i. grows towards its asymptotic value $\Delta f_{\max }$.

In Fig. 4 we show the conditional statistics and coherence interval of the estimator for the broadcast and downlink bursts. The simulation results are depicted with a dot notation, while the solid line represents the theoretical curve given by (14) and (16). The results for the uplink burst with short and long preamble and the direct link bursts are similar to those for the broadcast burst.

Broadcast Burst. In this case the maximum detectable offset $\Delta f_{\text {c.i. }}$ is seen to extend up to $0.42 \mathrm{MHz}=80 \mathrm{ppm}$ at $\mathrm{SNR}=0 \mathrm{~dB}$, and to $0.6 \mathrm{MHz}=115$ $\mathrm{ppm}$ at $\mathrm{SNR}=20 \mathrm{~dB}$. The estimator variance, normalized to $\left(2 \Delta f_{\max }\right)^{2}$ goes from $2.47 \cdot 10^{-3}$ at $\mathrm{SNR}=0 \mathrm{~dB}$ which corresponds to a standard deviation of about $62.1 \mathrm{kHz}=12 \mathrm{ppm}$, down to $1.5 \cdot 10^{-5}$ at $\mathrm{SNR}=20 \mathrm{~dB}$, with a standard deviation of about $4.8 \mathrm{kHz}=0.9 \mathrm{ppm}$.

Downlink Burst. In this case the estimation range, as was shown in Table 2 . is reduced. The c.i. is wider with respect to $\Delta f_{\max }$ than in the broadcast burst case, since more samples are used in the estimation. The maximum instability that can be correctly estimated extends up to $\pm 0.13 \mathrm{MHz}= \pm 25 \mathrm{ppm}$ at $\mathrm{SNR}$ $=0 \mathrm{~dB}$, and is raised to $\pm 0.15 \mathrm{MHz}= \pm 29 \mathrm{ppm}$ at $\mathrm{SNR}=20 \mathrm{~dB}$.

The statistics (14) and (16) imply a reliable prediction of the carrier frequency offset estimation conditional mean and variance at $\mathrm{SNR} \geq 10 \mathrm{~dB}$, as actually required by the hypothesis that led to their writing. The discrepancy between the theoretical and simulation results at low signal to noise ratio, which is particularly evident at $\mathrm{SNR}<5 \mathrm{~dB}$, derives from the heavy simplification introduced in Section 4.2.

\subsection{Performance of Symbol Timing Estimation with Time-Dispersive Channels}

Simulations were carried out with $h(t)$ channel models A and C, the power delay profiles of which are depicted in Fig. 5.

Channel A has a delay spread shorter than the guard interval, the transmitted signal stream is therefore not affected by the ISI caused by the dispersion of the channel impulse response. On the other hand, channel $\mathrm{C}$ has a higher delay spread than the guard interval. As an example of the estimators performance the results for uplink bursts with short preamble will be presented. 

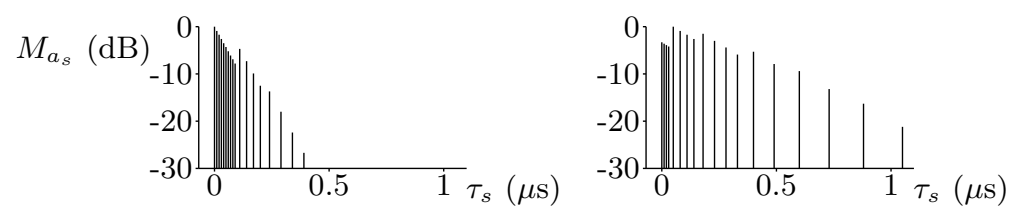

Fig. 5. Power delay profile of channel models A (left) and C (right)

We evaluate the symbol timing estimation performance by calculating the useful signal normalized power for each realization of the channel. The Cumulative Distribution Functions (CDFs) of $\bar{M}_{\mathrm{u}}$ and of the ratio $\bar{M}_{\mathrm{u}} /\left(\bar{M}_{\text {int }}+M_{w}\right)$, with $\bar{M}_{\text {int }}$ the normalized interference power and $M_{w}=2 \sigma^{2}$ the noise power are shown in Fig. 6. We observe that at $\mathrm{SNR}=5 \mathrm{~dB}$ in about $95 \%$ of the total simulated transmissions with channel model $\mathrm{A}$, the normalized useful power exceeds 0.9 and that in about only $5 \%$ of the realizations the signal to interference plus noise ratio is lowered by more than $0.5 \mathrm{~dB}$ with respect to the average channel $\mathrm{SNR}$ of $5 \mathrm{~dB}$. We also see that in the case of channel $\mathrm{C}$ the corresponding CDFs show a performance loss, since in about $90 \%$ of the transmissions, the normalized useful power exceeds 0.9 and in about $15 \%$ the signal to interference plus noise ratio falls under $4.5 \mathrm{~dB}$.
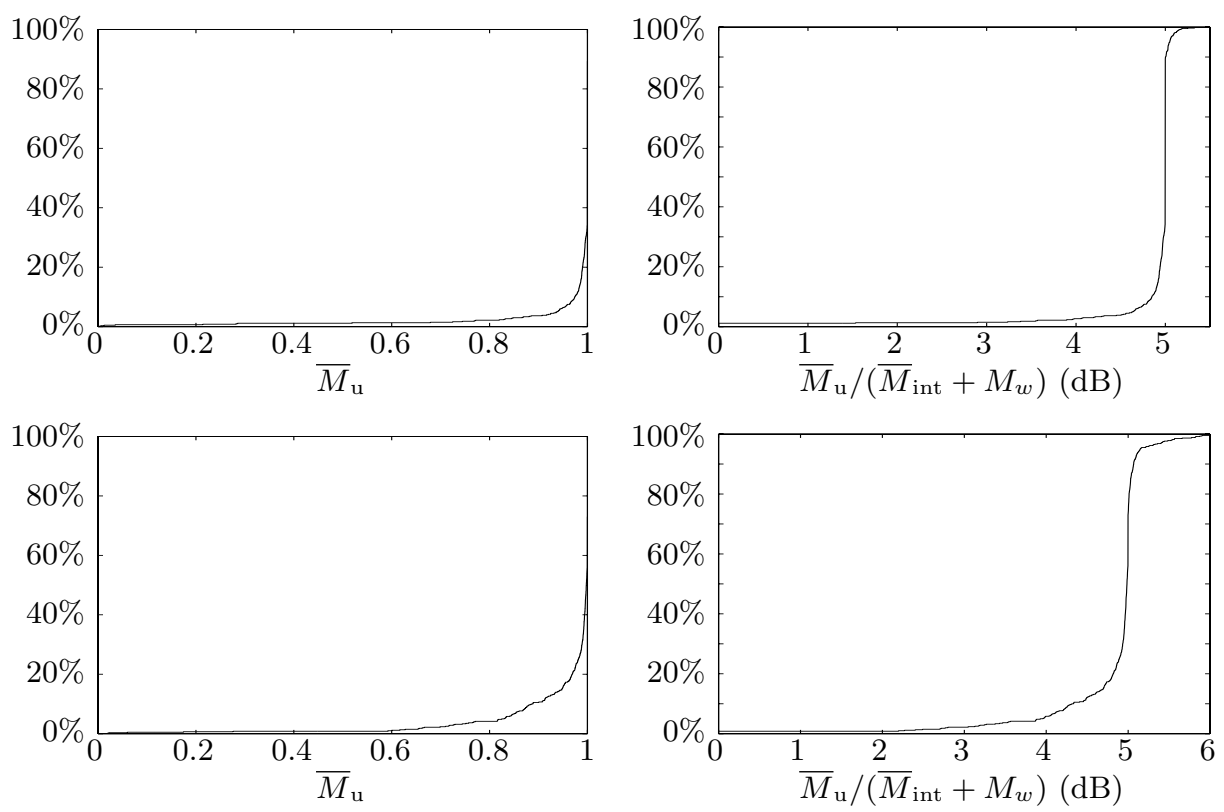

Fig. 6. Cumulative distribution functions of the normalized useful power (on the left) and of the signal to interference plus noise ratio (on the right) with channel models A (top) and C (bottom) 


\section{Conclusions}

The main objects of this work are time and frequency synchronization issues for ETSI Hiperlan/2 standard. Algorithms for time and frequency offset estimation, which are present in literature, have been adapted to all the operation modes provided by a Hiperlan/2 system. Their efficiency has been tested through the simulation of burst transmissions in the presence of either an AWGN or timedispersive channel impulse response.

A valuable technique for the evaluation of the performance of the symbol timing estimation in the presence of a time-dispersive channel has been proposed. It verifies the fraction of the useful power of the received signal after that the observation window has been placed on the OFDM symbol as indicated by the timing estimation algorithm.

An analytical description of the statistical properties of the carrier frequency offset estimation has been drawn under the hypothesis of high signal to noise ratio.

\section{References}

1. Broadband Radio Access Networks; HIgh Performance Radio Local Area Network (Hiperlan) Type 2; Requirements and Architectures for Wireless Broadband Access, ETSI Standard TR 101 031, ETSI, January 1999.

2. Broadband Radio Access Networks; Hiperlan Type 2; System Overview, ETSI Standard TR 101 683, ETSI, February 2000.

3. Broadband Radio Access Networks; Hiperlan Type 2; Physical (PHY) Layer, ETSI Standard TS 101 475, ETSI, February 2001.

4. L. Hanzo, W. Webb, T. Keller, Single- and Multicarrier Quadrature Amplitude Modulation: principles and applications for Personal Communications WLAN and Broadcasting, Wiley-IEEE Press, 2000.

5. T. Keller, L. Piazzo, P. Mandarini, L. Hanzo, "Orthogonal Frequency Division Multiplex Synchronization Techniques for Frequency-Selective Fading Channels", IEEE Journal on Selected Areas in Communications, vol. 19, no. 6, June 2001.

6. T.M. Schmidl, D.C. Cox, "Low-Overhead, Low Complexity [Burst] Synchronization for OFDM", Proceedings of 1996 IEEE International Conference on Communications, ICC '96, vol. 3, pp. 1301-1306.

7. T.M. Schmidl, D.C. Cox, "Robust Frequency and Timing Synchronization for OFDM", IEEE Transactions on Communications, vol. 45, no. 12, December 1997.

8. J. Medbo, P. Schramm, "Channel Models for Hiperlan/2 in Different Indoor Scenarios", ETSI EP BRAN 3ERIO85B, March 1998.

9. J. Medbo, "Radio Wave Propagation Characteristics at $5 \mathrm{GHz}$ with Modeling Suggestions for Hiperlan/2", ETSI EP BRAN WG3 Temporary document XX, January 1998.

10. N. Laurenti, Implementation Issues in OFDM Systems, Ph.D. thesis, Università di Padova, February 1999.

11. N.M. Blachman "Gaussian Noise-Part II: Distribution of Phase Change of NarrowBand Noise Plus Sinusoid", IEEE Transactions on Information Theory, 1988. 